Pacific Journal of Mathematic 


\title{
INTRINSIC TOPOLOGIES IN A TOPOLOGICAL LATTICE
}

\section{TAE Ho ChoE}

\begin{abstract}
It is shown that if $(L, T)$ is a compact connected modular topological lattice of finite dimension under a topology $T$, then the topology $T$, the interval topology of $L$, the complete topology of $L$, and the order topology of $L$ are all the same.
\end{abstract}

There are a variety of known ways in which a lattice may be given a topology, e.g., Frink's interval topology [8], Birkhoff's order topology [4], and Insel's complete topology [9].

A lattice $L$ is a topological lattice if and only if $L$ is a Hausdorff space in which the two lattice operations are continuous.

In this paper we give some of the relationships between topological lattice and its intrinsic topologies and extend a theorem of Dyer and Shields [7] and a result of Anderson [2]. We shall finally prove the main theorem stated above.

We shall use $A \wedge B$ and $A \vee B$ for a pair of subsets $A$ and $B$ of a lattice $L$ to denote the sets $\{a \wedge b \mid a \in A$ and $b \in B\}$ and $\{a \vee b \mid a \in A$ and $b \in B\}$, respectively. For a subset $A$ of $L, A^{*}$ is the closure of $A$. The empty set is written as

By the interval topology of a lattice $L$, denoted by $I(L)$, we mean the topology defined by taking the closed intervals $\{a \wedge L$, $a \vee L \mid a \in L\}$ as a sub-base for the closed sets. It is easy to see that if $(L, T)$ is a topological lattice and if $I(L)$ is Hausdorff, then $(L, T)$ is compact if and only if $T=I(L)$ and $L$ is complete.

For a net $\left\{x_{\alpha} \mid \alpha \in D\right\}$ in a complete lattice $L$, if $\lim \sup \left\{x_{\alpha} \mid \alpha \in D\right\}=$ $\lim \inf \left\{x_{\alpha} \mid x_{\alpha} \in D\right\}=x$, we say that the net $\left\{x_{\alpha}\right\}$ order converges to $x$. We define a subset $M$ of a complete lattice $L$ to be closed in the order topology of $L$, denoted by $O(L)$, if and only if no net in $M$ converges to a point outside of $M$.

The following two lemmas are immediate:

LEMma 1. If $(L, T)$ is a compact topological lattice, and if $\left\{x_{\alpha} \mid \alpha \in D\right\}$ is a monotone decreasing net in $L$ with $\inf \left\{x_{\alpha} \mid \alpha \in D\right\}=a$, then the net converges to $a$ in $T$. The dual argument is also true.

Lemma 2. If $(L, T)$ is a compact topological lattice, then $T \subset O(L)$. Moreover, if $O(L)$ is also compact, then $T=O(L)$.

By a complete subset $C$ of a lattice $L$ we shall mean a nonempty subset $C$ of $L$ such that for each nonempty subset $S$ of $C, S$ possesses both a sup $S$ and an inf $S$ in $L$, and furthermore, both sup $S$ and 
inf $S$ are in $C$. The smallest topology for $L$ in which the complete subsets of $L$ are closed is called the complete topology for $L$, and denoted by $C(L)$. It is known [9] that $C(L) \subset O(L)$, and if $L$ is complete, then $I(L) \subset C(L)$.

The following lemma follows at once either from Lemmas 1 and 2 or from [11].

LEMmA 3. If $(L, T)$ is a compact topological lattice, then $I(L)$ is Hausdorff, if and only if $I(L)=C(L)=T=O(L)$.

The breadth of a lattice $L$ is the smallest integer $n$ such that any finite subset $F$ of $L$ has a subset $F^{\prime \prime}$ of at most $n$ elements such that $\inf F=\inf F^{\prime \prime}$. It is known [4] that the breadth of $L$ is equal to the breadth of the dual of $L$.

A subset $M$ of a topological lattice $L$ is convex if and only if $(M \wedge L) \cap(M \vee L)=M$ [1]. A topological lattice is locally convex if and only if the convex open sets form a basis for the topology. It is well known that a compact (or locally compact and connected) topological lattice is locally convex.

We shall extend a theorem of Dyer and Shields in [7] as follows:

THeOREM 1. If $L$ is a locally compact, locally convex topological lattice of finite breadth and $U$ is a neighborhood of a point $x$ in $L$, then there exist two elements $y$ and $z$ in $L$ and a neighborhood $V$ of $x$ such that $V \subset[y, z] \subset U$.

Proof. Choose neighborhoods $U_{0}, U_{1}$ and $U_{2}$ of $x$ such that $U_{0}$ and $U_{2}$ are convex, $U_{1}^{*}$ compact, and $U_{0} \subset U_{1}^{*} \subset U_{2} \subset U$. Again we can choose two neighborhood $U_{3}$ and $U_{4}$ of $x$ such that $U_{3} \wedge \cdots \wedge U_{3}$ ( $n$ times) $\subset U_{0}$ and $U_{4} \vee \cdots \vee U_{4}(n$ times $) \subset U_{0}$, where $n$ is the breadth of $L$. Setting $V=U_{3} \cap U_{4}$ we consider the sublattice $W$ of $L$ generated by $V$. Since every element $w$ of $W$ can be expressed as a lattice-polynomial of finitely many elements $x_{1}, x_{2}, \cdots, x_{m}$ of $V$, we have inf $x_{i} \leqq w \leqq \sup x_{i}$. Suppose $m>n$. By definition of breadth we can choose at most $n$ elements $x_{i}^{\prime}$ from those $x_{i}^{\prime}$ s such that inf $x_{i}=\inf x_{i}^{\prime}$. Thus inf $x_{i} \in U_{0}$. Similarly, sup $x_{i} \in U_{0}$. Clearly $W \subset U_{0}$ and $W^{*} \subset U_{1}^{*}$. Since $W^{*}$ is a compact sublattice, $W^{*}$ has a maximal element $z$ and a minimal element $y$. Now consider the smallest convex subset $\boldsymbol{C}\left(W^{*}\right)=\left(W^{*} \wedge L\right) \cap\left(W^{*} \vee L\right)$ containing $W^{*}$ in $L$ (see [1]). It is easy to see that $C\left(W^{*}\right)=[y, z]$. And $V \subset[y, z] \subset U_{2} \subset U$. The proof is complete.

Since compactness implies local convexity in a topological lattice, the distributivity hypothesis in Theorem 3 in [7] is not necessary. 
It is remarked that the hypothesis of finite breadth in Theorem 1 can be replaced by finite dimension. The author, however, does not know how to obtain this result without using connectedness. For example, the space $2^{X}(X$ is an infinite set) has infinite breadth, but has zero dimension. And we note that the $2^{x}$ is Hausdorff in its interval topology [10]. (See [4], Problem 81).

A topological lattice is chain-wise connected if and only if for each pair of elements $x$ and $y$ with $x \leqq y$ there is a closed connected chain from $x$ to $y$. It is well known [12] that a locally compact connected topological lattice is chain-wise connected.

We shall show that the hypothesis of distributivity in Anderson's result ([2], Corollary 1) can be replaced by modularity. The proof is essentially the same as in [2].

LEMMA 4. If $L$ is a locally compact connected modular topological lattice, then the breadth of $L$ is less than or equal to the codimension of $L$.

Proof. Suppose the codimension of $L$ is $n$. If the breadth of $L$ is $\$ n$, then $L$ contains an $n+1$ element subset $A$, say $A=$ $\left\{x_{1}, \cdots, x_{n+1}\right\}$, such that $\inf A \neq \inf B$ for any proper subset $B$ of $A$. Let $b_{i}=\inf \left(A \backslash x_{i}\right), i=1,2, \cdots, n+1$, and let $a=\inf A$. Then $b_{i} \neq a$, $i=1, \cdots, n+1$, and $b_{i} \neq b_{j}(i \neq j)$. Let $I_{i}$ be the closed interval $\left[a, b_{i}\right], i=1,2, \cdots, n+1$. Now consider two mappings

$$
f: I_{1} \times \cdots \times I_{n+1} \rightarrow I_{1} \vee \cdots \vee I_{n+1} \subset L
$$

defined by $f\left(a_{1}, \cdots, a_{n+1}\right)=a_{1} \vee \cdots \vee a_{n+1}$, and $g: I_{1} \vee \cdots \vee I_{n+1} \rightarrow$ $I_{1} \times \cdots \times I_{n+1}$ defined by $g\left(a_{1} \vee \cdots \vee a_{n+1}\right)=\left(b_{1} \wedge\left(a_{1} \vee \cdots \vee a_{n+1}\right)\right.$, $\left.\cdots, b_{n+1} \wedge\left(a_{1} \vee \cdots \vee a_{n+1}\right)\right)$. Then clearly $f$ and $g$ are well defined and continuous. Furthermore, $f^{-1}=g$, because by modularity we have

$$
\begin{aligned}
a_{1} & \leqq b_{1} \wedge\left(a_{1} \vee \cdots \vee a_{n+1}\right)=a_{1} \vee\left(b_{1} \wedge\left(a_{2} \vee \cdots \vee a_{n+1}\right)\right) \\
& \leqq a_{1} \vee\left(b_{1} \wedge\left(b_{2} \vee \cdots \vee b_{n+1}\right)\right) \leqq a_{1} \vee\left(b_{1} \wedge x_{1}\right)=a_{1} \vee a=a_{1},
\end{aligned}
$$

and hence $b_{1} \wedge\left(a_{1} \vee \cdots \vee a_{n+1}\right)=a_{1}$, and similarly for $i=2, \cdots, n+1$.

On the other hand, since such $I_{i}$ is locally compact and connected in its relative topology, $I_{i}$ contains a nondegenerate compact connected chain $C_{i}, i=1,2, \cdots, n+1$. The subset $C_{1} \times \cdots \times C_{n+1}$ of $I_{1} \times \cdots \times$ $I_{n+1}$ has codimension $n+1$ [6]. Hence, the codimension of the closed subset $f\left(C_{1} \times \cdots \times C_{n+1}\right)$ of $L$ is $n+1$. We thus have a contradiction.

Lemma 5. If $(L, T)$ is a compact topological lattice of finite breadth, then $I(L)$ is Hausdorff.

Proof. For two distinct elements $x$ and $y$ of $L$, choose $T$-neigh- 
borhoods $U$ and $V$ of $x$ and $y$, respectively, such that $U \cap V=\square$. For each element $z$ of $L \backslash\{x, y\}$, choose $a T$-neighborhood $W$ of $z$ such that $W \cap\{x, y\}=\square$. By Theorem 1, we can find $T$-neighborhoods $U^{\prime}, V^{\prime}$ and $W^{\prime}$ of $x, y$ and $z$, respectively, and closed intervals $\left[x_{1}, x_{2}\right]$, $\left[y_{1}, y_{2}\right]$ and $\left[z_{1}, z_{2}\right]$ such that $U^{\prime} \subset\left[x_{1}, x_{2}\right] \subset U, V^{\prime} \subset\left[y_{1}, y_{2}\right] \subset V$ and $W^{\prime} \subset\left[z_{1}, z_{2}\right] \subset W$. Clearly the family $\mathscr{W}=\left\{U^{\prime}, V^{\prime}, W^{\prime} \mid z \in L \backslash\{x, y\}\right\}$ is an open covering of $L$. So there is a finite sub-family of $\mathscr{W}$ which covers $L$. Therefore, there is a finite family of closed intervals whose union is $L$ such that no interval contains both $x$ and $y$. It follows by Proposition 1 in [10] that $I(L)$ is Hausdorff.

Summarizing Lemmas 4,5 and 3, we have the following main theorem:

THEOREM 2. If $(L, T)$ is a compact, connected, modular topological lattice of finite codimension, then $I(L)=C(L)=T=O(L)$.

COROLlaRY 1. If $(L, T)$ is a compact topological lattice of finite. breadth, then $I(L)=C(L)=T=O(L)$.

\section{REFERENCES}

1. L. W. Anderson, On one dimension topological lattices, Proc. Amer. Math. Soc. 10 (1959), 715-720.

2. On the breadth and codimension of topological lattices, Pacific J. Math. 9 (1959), 327-333.

3. - On the distributivity and simple connectivity of plane lattices, Trans. Amer. Math. Soc. 91 (1959), 102-112.

4. G. Birkhoff, Lattice theory, Rev. Ed., Amer. Math. Soc. Coll., (1967).

5. T. H. Choe, On compact topological lattices of finite dimension, to appear, Trans. Amer. Math. Soc.

6. H. Cohen, A cohomological definition of dimension for locally compact Hausdorff spaces, Duke Math. J. 21 (1954), 209-224.

7. E. Dyer and A. Shields, Connectivity of topological lattices, Pacific J. Math. 9 (1959).

8. O. Frink, Topology in lattices, Trans. Amer. Math. Soc. 15 (1942), 569-582.

9. Arnold J. Insel, $A$ relationship between the complete topology and the order topology. of a lattice, Proc. Amer. Math. Soc. 15 (1964), 849-850.

10. E. S. Northam, The interval topology of a lattice, Proc. Amer. Math. Soc. 4 (1953), 824-829.

11. A. J. Ward, On relations between certain intrinsic topologies in partially ordered sets, Proc. Cam. Phil. Soc. 51 (1955), 254-261.

12. L. W. Ward. Jr. and L. W. Anderson, A structure theorem for topological lattices, Proc. Glasgow Math. Assoc. 5 (1961), 1-3.

Received November 3, 1967,

UNIVERSITY OF MASSACHUSETTS AND

MCMASTER UNIVERSITY 


\section{PACIFIC JOURNAL OF MATHEMATICS}

\section{EDITORS}

H. ROYDEN

Stanford University

Stanford, California

R. R Phelps

University of Washington

Seattle, Washington 98105
J. DUGUNDJI

Department of Mathematics

University of Southern California

Los Angeles, California 90007

RICHARD ARENS

University of California

Los Angeles, California 90024

\section{ASSOCIATE EDITORS}
E. F. BECKENBACH
B. H. NeumanN
F. WOLF
K. YosidA

\section{SUPPORTING INSTITUTIONS}

UNIVERSITY OF BRITISH COLUMBIA

CALIFORNIA INSTITUTE OF TECHNOLOGY

UNIVERSITY OF CALIFORNIA

MONTANA STATE UNIVERSITY

UNIVERSITY OF NEVADA

NEW MEXICO STATE UNIVERSITY

OREGON STATE UNIVERSITY

UNIVERSITY OF OREGON

OSAKA UNIVERSITY

UNIVERSITY OF SOUTHERN CALIFORNIA
STANFORD UNIVERSITY

UNIVERSITY OF TOKYO

UNIVERSITY OF UTAH

WASHINGTON STATE UNIVERSITY

UNIVERSITY OF WASHINGTON

AMERICAN MATHEMATICAL SOCIETY CHEVRON RESEARCH CORPORATION TRW SYSTEMS

NAVAL WEAPONS CENTER 


\section{Pacific Journal of Mathematics

Vol. 28, No. $1 \quad$ March, 1969

Patrick Robert Ahern, On the geometry of the unit ball in the space of real annihilating measures .............................. 1

Kirby Alan Baker, Equational classes of modular lattices ............. 9

E. F. Beckenbach and Gerald Andrew Hutchison, Meromorphic minimal surfaces ......................................... 17

Tae Ho Choe, Intrinsic topologies in a topological lattice ..............

John Bligh Conway, A theorem on sequential convergence of measures and

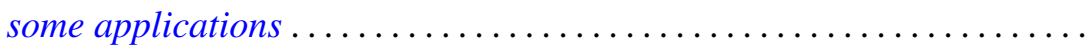

Roger Cuppens, On the decomposition of infinitely divisible probability laws without normal factor.

Lynn Harry Erbe, Nonoscillatory solutions of second order nonlinear

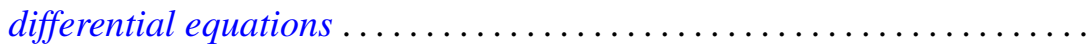

Burton I. Fein, The Schur index for projective representations of finite

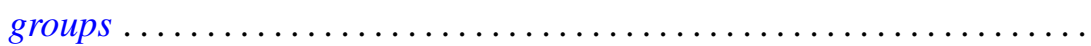

Stanley P. Gudder, A note on proposition observables............... 101

Kenneth Kapp, On Croisot's theory of decompositions ............... 105

Robert P. Kaufman, Gap series and an example to Malliavin's theorem . . . 117

E. J. McShane, Robert Breckenridge Warfield, Jr. and V. M. Warfield,

Invariant extensions of linear functionals, with applications to measures and stochastic processes ................

Marvin Victor Mielke, Rearrangement of spherical modifications ...

Akio Osada, On unicity of capacity functions ..............

Donald Steven Passman, Some 5/2 transitive permutation groups ...

Harold L. Peterson, Jr., Regular and irregular measures on groups and dyadic spaces...

Habib Salehi, On interpolation of $q$-variate stationary stochastic processes...

Michael Samuel Skaff, Vector valued Orlicz spaces generalized

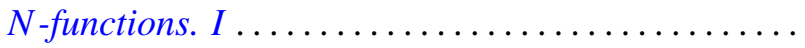

A. J. Ward, On $H$-equivalence of uniformities. II...........

Thomas Paul Whaley, Algebras satisfying the descending chain condition for subalgebras...

G. K. White, On subgroups of fixed index

Martin Michael Zuckerman, A unifying condition for implications among the axioms of choice for finite sets ................. 